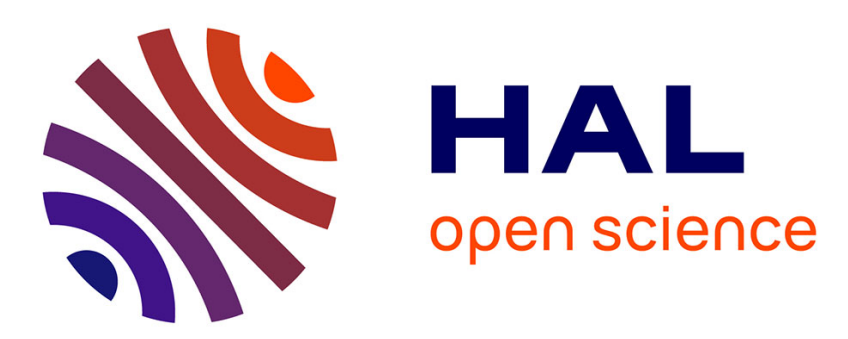

\title{
Consommation d'énergie et théorie des pratiques : vers des pistes d'action pour la transition énergétique
} Patricia Roques, Dominique Roux

\section{To cite this version:}

Patricia Roques, Dominique Roux. Consommation d'énergie et théorie des pratiques: vers des pistes d'action pour la transition énergétique. Décisions Marketing, 2018, 90 (2), pp.35-54. 10.7193/DM.090.35.54 . hal-02022162

\section{HAL Id: hal-02022162 \\ https://hal.science/hal-02022162}

Submitted on 5 Jul 2021

HAL is a multi-disciplinary open access archive for the deposit and dissemination of scientific research documents, whether they are published or not. The documents may come from teaching and research institutions in France or abroad, or from public or private research centers.
L'archive ouverte pluridisciplinaire $\mathbf{H A L}$, est destinée au dépôt et à la diffusion de documents scientifiques de niveau recherche, publiés ou non, émanant des établissements d'enseignement et de recherche français ou étrangers, des laboratoires publics ou privés. 


\title{
Consommation d'énergie et théorie des pratiques : d'une typologie de profils aux pistes d'action pour la transition énergétique
}

\author{
Patricia Roques et Dominique Roux
}

\section{Patricia Roques}

Docteur en Sciences de gestion

Université Nice Sophia-Antipolis-CNRS UMR 7321

GREDEG

250 rue A. Einstein, Sophia Antipolis, Bât. 2

06560 Valbonne, France

Tél : 0621426535

patricia.roques@unice.fr

\section{Dominique Roux}

Professeure de marketing

Université de Reims Champagne-Ardenne

UFR des Sciences Economiques, Sociales et de Gestion

Laboratoire Regards, EA 6292

Bâtiment Recherche

57 bis rue Pierre Taittinger

51096 Reims cedex

Tél : 0688261035

dominique.roux@univ-reims.fr

- Pour citer cet article : Roques P. et Roux D. (2018), Consommation d'énergie et théorie des pratiques : vers des pistes d'action pour la transition énergétique, Décisions Marketing, 90: $35-54$. 


\title{
Consommation d'énergie et théorie des pratiques : d'une typologie de profils aux pistes d'action pour la transition énergétique
}

\begin{abstract}
Résumé
Dans un contexte d'incitation à la réduction énergétique, quels sont les leviers d'action sur les comportements de consommation des individus, au-delà des valeurs qu'ils associent à la maîtrise de leur consommation ? Sur la base d'une étude qualitative menée avec 40 répondants d'un même complexe locatif réhabilité, cette recherche mobilise la théorie des pratiques appliquée au contexte de la consommation énergétique. Après avoir présenté et discuté ce cadre d'analyse peu usité en marketing, elle propose une typologie de profils. Ceux-ci intègrent à la fois les savoir-faire, les routines et les significations attachées à un ensemble d'activités consommatrices d'énergie, mais aussi le système d'équipement incorporé au logement et les incitations/prescriptions qui ont accompagné le programme de réhabilitation énergétique. Les résultats enrichissent les approches comportementales par la prise en compte de l'environnement socio-technique des individus et fournissent une série de recommandations utiles aux bailleurs sociaux comme aux organisations publiques ou marchandes engagées dans ces problématiques. Mots clés : consommation d'énergie, théorie des pratiques, efficacité énergétique, sobriété, réhabilitation thermique de l'habitat social.

\section{Energy consumption and practice theory: From a consumer typology to possible actions for energy transition}

\begin{abstract}
In a context where of energy reduction is needed, what are levers for action on individuals' consumption patterns beyond values associated to the mastering of their energy use? On the basis of a qualitative study carried out with 40 informants from the same rehabilitated rental complex, this research first proposes a theorization of energy practices. After having presented and discussed the practice-theory framework still new in the marketing field, we propose a typology of profiles. These incorporate the knowhow, routines and meanings associated with a set of energy-consuming activities, but also the system of equipment incorporated in housing and the incentives/prescriptions that accompanied the program of energy rehabilitation. The results enrich behavioral approaches by taking into account individuals' socio-technical environment and provide a series of useful recommendations to social landlords as well as public and commercial organizations involved in these issues.
\end{abstract}

Key words: energy consumption, practice theory, energy efficiency, sobriety, energy rehabilitation of social housing. 
La question de la transition énergétique est au cœur des débats et des politiques publiques qui considèrent comme urgentes la transformation des modes de vie et leur réorientation vers des logiques de soutenabilité (Bourg et alii, 2016). Réduire la demande d'énergie constitue en effet un enjeu majeur en lien avec l'épuisement ou la rareté des ressources, et de façon cruciale, avec la problématique du changement climatique. Celle-ci renforce l'impératif d'une diminution de la demande en énergie ${ }^{1}$, dans laquelle le secteur résidentiel représente $27 \%$ de la consommation totale en France $^{2}$, soit presque autant que les transports et davantage que l'industrie. Aussi, des politiques publiques, contraignantes ou incitatives, ont été mises en œuvre dès 1974 à la suite des chocs pétroliers, avec l'objectif principal d'accroître l'efficacité énergétique des logements. Désormais, la transition énergétique fait 1 'objet d'une $10 i^{3}$ qui vise à lutter contre le dérèglement climatique, comme à renforcer l'indépendance énergétique du pays. Y sont favorisées les mesures susceptibles d'encourager les économies d'énergie par la rénovation des logements, et incitant par ailleurs les individus à se doter d'équipements efficaces. Pourtant, en dépit des efforts accomplis et poursuivis à marche forcée, l'Agence Européenne pour l'Environnement (AEE) soulignait récemment que les deux tiers des gains liés à l'efficacité énergétique sur les vingt dernières années ont été contrebalancés par des augmentations de consommation, liées notamment à la multiplication des équipements et des usages, phénomène connu sous le nom d' «effet rebond $»^{4}$. La réduction de la consommation d'énergie, obtenue par une plus grande efficacité et un meilleur rendement des équipements, semble donc insuffisante à elle seule pour atteindre les objectifs de réduction fixés à l'horizon 2050. Elle appelle donc pour certains un engagement plus radical vers la sobriété, consistant à redimensionner, limiter, réduire ou mutualiser les équipements (Bourg et alii, 2016). De plus, alors que deux personnes sur trois déclarent s'imposer régulièrement des restrictions sur plusieurs postes budgétaires et que les gains de pouvoir d'achat des ménages $(+1,6 \%$ par rapport

\footnotetext{
${ }^{1}$ Voir les travaux de l'Agence Internationale de l'Energie IEA/AIE et les conclusions de la COP 21, 21ème Conférence des Parties sur le climat, conférence des Nations Unies sur les changements climatiques, tenue à Paris du 30 novembre au 11 décembre 2015.

2 ADEME (2016), L’efficacité énergétique en Europe, http://www.connaissancedesenergies.org/sites/default/files/pdf-pt-vue/efficaciteenergetiqueeneurope.pdf ${ }^{3}$ La loi de transition énergétique pour la croissance verte a été publiée au JO du 18 août 2015.

${ }^{4}$ European Environment Agency (2015), Progress on energy efficiency in Europe, Odyssee energy efficiency index (ODEX), EU28, p.14, http://www.eea.europa.eu/data-and-maps/indicators/progress-onenergy-efficiency-in-europe-2/assessment-1
} 
à 2016) sont érodés par l'inflation (+1\%) provoquée par la hausse des prix de l'énergie ${ }^{5}$, les individus continuent à investir dans l'acquisition d'équipements électriques ainsi que dans les abonnements (téléphonie, télévision payante, Internet) qui leur sont liés ${ }^{6}$. De fait, les changements de comportements des individus semblent très insuffisants en dépit de leurs attitudes favorables ou des valeurs qu'ils associent aux économies d'énergie (Innocent, François-Lecompte et Le Gall-Ely, 2016 ; Zélem, 2010). Brisepierre (2011) confirme également que la sensibilité écologique n'entraîne pas à elle seule plus de sobriété énergétique, ni de changement radical dans les pratiques qui chacune fonctionne selon des logiques différentes. Par exemple, consommer de l'énergie pour se chauffer n'est pas similaire au fait d'en user pour jouer sur un ordinateur ou pour cuisiner de manière récréative. Réussir la transition énergétique passe donc par une compréhension plus fine de l'ensemble des pratiques de consommation affectant de manière positive ou négative la consommation énergétique, mais également des normes sociales et de l'environnement matériel dans lesquels elles s'insèrent (Moloney et Strengers, 2014). L'objectif de cette recherche est donc de proposer une typologie d'individus basée sur un ensemble de pratiques qualifiées selon leur caractère favorable ou défavorable à la réduction de leur consommation énergétique. Cette typologie intègre l'environnement dans lequel les individus sont placés, c'est-à-dire l'infrastructure matérielle (les installations du logement et les divers appareils acquis) ainsi que les normes sociales que sont par exemple la température de chauffage recommandée d'un logement, les « bons gestes » évitant la déperdition de chaleur, le temps nécessaire pour une douche, etc. (Shove, 2003). Nous mobilisons pour cela la théorie des pratiques (Reckwitz, 2002 ; Schatzki, 1996, 2002 ; Warde, 2005 ; Shove et Pantzar, 2005) qui considère les comportements, énergétiques en l'occurrence, comme la résultante plus ou moins routinisée d'un ensemble indissoluble de «significations, de compétences et d'objets » qui constituent les dimensions centrales de la pratique (Dubuisson-Quellier et Plessz, 2013). La théorie des pratiques prolonge l'approche des coûts/bénéfices attachés à certaines facettes sociales, hédoniques, utilitaires, cognitives, expressives et éthiques de la maîtrise de la consommation électrique (Innocent, François-Lecompte et Le Gall-

\footnotetext{
${ }^{5}$ http://www.lefigaro.fr/conjoncture/2017/10/05/20002-20171005ARTFIG00257-1-insee-releve-a-18-lacroissance-de-2017.php

${ }^{6}$ CREDOC (2013), Enquête «Conditions de vie et Aspirations des Français », La diffusion des technologies de l'information et de la communication dans la société française, $\mathrm{n}^{\circ} 297$. http://www.arcep.fr/uploads/tx_gspublication/rapport-CREDOC_2013-dec2013.pdf
} 
Ely, 2016), en ré-enchâssant ces valeurs et comportements dans le contexte social, technologique et institutionnel qui les façonne (Stephenson et alii, 2010). A l'instar de Dujin, Maresca et Vedie (2012), elle propose de considérer la manière dont ces usages, appréhendés dans leur épaisseur collective, épousent (ou non) les hypothèses comportementales dont sont porteurs les programmes étudiés et servent (ou non) les objectifs de réduction définis par les acteurs institutionnels.

Les pratiques ont été analysées par le biais d'une étude qualitative et par observations menée auprès de 40 répondants d'un ensemble de logements sociaux locatifs de la région Provence-Alpes-Côte d'Azur ayant fait l'objet du même programme de réhabilitation thermique, incluant un programme d'accompagnement et de sensibilisation des usagers. Ce programme, soumis au volontariat et suivi par un tiers de notre échantillon, comportait un volet collectif avec des réunions d'information et d'échanges entre participants sous la forme d'ateliers et un volet individualisé avec des visites au domicile. Pour éviter tout biais lié à des différences socio-économiques marquées dans l'échantillon, et donc susceptibles d'entacher la comparabilité des résultats (Gram-Hanssen, 2008), la recherche a été menée auprès d'une population homogène placée dans des conditions similaires, c'est-à-dire confrontée par nécessité (et non par choix) au même programme de réhabilitation énergétique. Un inventaire du système technique incorporé au logement, soit l'ensemble des dispositifs tels que chaudières, radiateurs, chauffe-eau et thermostats nécessaires à la régulation de la température, a été réalisé. Il a été complété par une phase d'entretiens qualitatifs visant à comprendre les activités des individus, les buts qu'ils poursuivent, le sens qu'ils donnent à leur pratiques et à leur consommation d'énergie, et les règles auxquelles ils se conforment ou non, en suivant les cinq dimensions identifiées par Gram-Hanssen (2008) dans le contexte de la consommation énergétique. L'analyse de ce matériau a permis de distinguer quatre profils : les «volontaires », les « responsables », les « empêchés » et les «réfractaires ». Notre approche présente deux contributions. Premièrement, elle enrichit la théorisation des pratiques dans le domaine de l'énergie (Gram-Hanssen, 2008 ; Stephenson et alii, 2010) en tenant compte d'un large éventail d'activités susceptibles d'impacter la consommation énergétique, notamment par l'introduction croissante d'appareils électriques et électroniques additionnels dans le logement qui consomment de grandes quantités d'« énergie grise », engendrent de forts impacts 
environnementaux (terres rares, obsolescence rapide, déchets) et induisent des consommations externes non comptabilisées au titre des consommations résidentielles (réseaux, serveurs). Deuxièmement, en qualifiant la valence de ces comportements favorables ou défavorables à la réduction de la consommation énergétique -, cette recherche fournit aux acteurs institutionnels et aux bailleurs sociaux une typologie de profils reflétant leur aptitude au changement. De plus, appuyée sur la théorie des pratiques, cette typologie met au jour les dimensions les plus discriminantes selon les profils, facilitant ainsi pour chacun l'identification des leviers d'action possibles.

\section{La consommation d'énergie à la lumière de la théorie des pratiques}

Dans la littérature, de multiples approches ont été mobilisées pour comprendre les déterminants de la consommation énergétique (Innocent, François-Lecompte et Le GallEly, 2016 ; Lutzenhiser, 1992 ; Stephenson et alii, 2010 ; Wilson et Dowlatabadi, 2007). Comme le montrent ces auteurs, les recherches empruntent à toutes les disciplines, de la psychologie environnementale à la sociologie, en passant par la micro-économie et l'économie comportementale (Dujin, Maresca et Vedie, 2012). Les modèles psychologiques visant à expliquer ou prédire les comportements ayant montré leurs limites, des travaux ont proposé des approches systémiques de la «sociologie de l'énergie ». Les premiers travaux ont développé la notion de «cultures de l'énergie » (Lutzenhiser, 1992) en se référant aux styles de vie des individus. Ce faisant, ils visaient à combler le fossé identifié par Wilson et Dowlatabadi (2007) entre des traditions de recherche en économie comportementale exclusivement centrées sur l'individu et celles portant plus largement sur 1'habitus comme trace des structures sociales (Bourdieu, 1980). La théorie des pratiques initiée par Schatzki (1996) et Reckwitz (2002) permet de réconcilier les approches micro/macro en considérant que les dimensions cognitives et symboliques de la consommation sont une expression directe du social, incarnée dans des routines : "Une pratique est un type de comportement routinisé qui se compose de plusieurs éléments, interconnectés les uns aux autres : les formes d'activités corporelles, les formes d'activités mentales, les «choses» et leur utilisation, les connaissances de base sous forme de compréhension, de savoir-faire, d'états émotionnels et de motivations »(Reckwitz, 2002, p. 249). La théorie des pratiques vise ainsi à saisir les comportements comme «performances observables » et entrelacs 
d'activités et de représentations qui sont guidés par des buts, façonnés par des normes (Schatzki, 2002) et enchâssés dans un environnement matériel particulier (Reckwitz, 2002 ; Warde, 2005). La théorie des pratiques considère que ces comportements ne sont pas produits par des acteurs sociaux purement rationnels, mais continuellement recréés par eux en tant qu'acteurs (Giddens, 1984). La théorie des pratiques dont les approches ne sont pas toutes convergentes ou stabilisées (encadré 1) a été plus directement adaptée au domaine de la consommation énergétique, en particulier le confort thermique (GramHanssen, 2008). Son apport est de considérer les différentes dimensions constitutives des pratiques, au-delà d'une approche anthropocentrée (dirigée sur l'individu et ignorant son environnement matériel) et purement délibérative (centrée sur le caractère prédictif des discours déclarés, en ignorant le poids des routines incorporées, des structures et des contraintes). En raison de sa pertinence pour le domaine étudié (Dubuisson-Quellier et Plessz, 2013), cette approche a été privilégiée dans cette recherche. Dans la continuité de Warde (2005) et Schatzki (1996, 2002), Gram-Hanssen (2008) analyse les routines de régulation thermique dans un contexte résidentiel homogène ayant bénéficié d'un programme d'affichage environnemental. De Warde (2005), elle retient les quatre dimensions d'analyse des pratiques: les formes de compréhension de la manière de faire les choses, le niveau d'engagement des individus dans leurs pratiques, les procédures (ou normes de comportements) qu'ils partagent autour du «faire» et du « dire », et les objets présents dans leur consommation. Sur la première dimension, elle conserve toutefois le découpage opéré par Schatzki (2002) entre la «compréhension pratique »-c'est-à-dire les routines physiques et mentales incorporées par les individus - et "l'intelligibilité pratique », autrement dit le sens qu'ils donnent à ce qu'ils font. Son modèle intègre les règles explicites et les savoirs tacites qui font d'une grande partie des activités domestiques des tâches routinières auxquelles les individus sont capables de prêter un sens. Il en va ainsi de l'extinction de l'éclairage dans les pièces inoccupées dans l'intention, tacite, de ne pas gaspiller. Considérant aussi la dimension matérielle de la consommation, son modèle prend en compte l'influence de la technologie induisant certaines conduites (par exemple, un thermostat délègue aux appareils le réglage de la température, un «compteur intelligent» complété d'un dispositif de feed back vise à informer les individus de leur consommation pour les inciter à la réduire) (Dujin, Maresca et Vedie, 2012). Toutefois, si Gram-Hanssen 
(2008) met au jour des différences dans les comportements de régulation thermique de ses interviewé(e)s, elle ne saisit pas l'impact d'autres pratiques (Moloney et Strengers, 2014), par exemple faire la lessive ou la cuisine, travailler ou se distraire, qui impliquent elles-mêmes des modes d'usage du système incorporé au logement, mais aussi l'achat et l'utilisation de matériels complémentaires (appareils électro-ménager, équipements informatiques, chauffages d'appoint, etc.). De plus, Gram-Hanssen (2008) ne fait pas apparaître ce qui pourrait être favorable ou défavorable à la réduction de la consommation énergétique des individus, deux limites que cette recherche se propose de combler pour appréhender plus efficacement les leviers de réduction de la consommation énergétique. Notre approche consiste à analyser comment un ensemble de pratiques et d'éléments cognitifs, normatifs, et matériels qui les entourent interagissent (Moloney et Strengers, 2014), et selon quels critères leur hétérogénéité peut être comprise en dépit d'une relative uniformité des conditions de vie.

Encadré 1 : Une synthèse des recherches sur la théorie des pratiques

En dépit de sa qualité d'intégration des niveaux micro et macro, la théorie des pratiques n'est pas homogène (Dubuisson-Quellier et Plessz, 2013). Epistémologiquement d'abord, les approches anglo-saxonnes (Schatzki, 1996, 2002 ; Reckwitz, 2002) voient dans les pratiques des formes de réalisation du social couplées à des arrangements matériels. Différemment, la praxéologie de Bourdieu (1980) cherche plutôt à comprendre le mode de reproduction des pratiques tandis que la sociologie de l'acteurréseau constitue une troisième voie, orthogonale à la précédente, dans laquelle les pratiques sont des points d'entrée pour analyser la constitution des faits stabilisés. Empiriquement ensuite, les dimensions retenues pour caractériser les pratiques ne sont pas identiques d'un chercheur à l'autre. Schatzki (2002) n'incorpore ni la technologie ni la culture matérielle, alors que celles-ci sont des éléments centraux dans le «tournant non-humain » qui donne aux objets une agence symétrique à celle des acteurs (Shove et Pantzar, 2005 ; Warde, 2005). La difficulté d'opérationnalisation du cadre théorique proposé initialement par Reckwitz (2002) aboutit également à des propositions de simplification, celle de Warde (2005) ne considérant que quatre éléments et celle de Shove et Pantzar (2005) agrégeant les normes et les formes de compréhension de l'activité sous la notion globale de «compétences » (Tableau 1).

En raison de son adéquation au domaine de l'énergie, nous nous référons ici au cadre de la théorie des pratiques proposé par Gram-Hanssen (2008) qui dégage cinq dimensions : 1. le degré de compréhension pratique et d'appropriation des éléments incorporés ou acquis (routines d'usage des appareils, gestion de leur mise en veille, manipulation des robinets thermostatiques, etc.)

2. l' « intelligibilité pratique » et les raisons d'être des routines incorporées : il s'agit de saisir les justifications et les rationalisations données par les individus pour expliquer ce qu'ils font dans leurs activités quotidiennes. 
3. les engagements et significations accordées aux pratiques : ces dimensions se rattachent au désir et au sens donné à ce qui est fait, par exemple chauffer ou améliorer le confort de son logement, réaliser des travaux d'isolation ou d'embellissement, acheter des équipements de loisirs, s'extraire du logement pour d'autres activités moins énergivores (comme jouer dehors, faire du sport ou fréquenter une bibliothèque).

4. les règles, prescriptions et influences («Rules, Knowledge, langage », GramHanssen, 2008) : elles proviennent des sources proximales ou distales auxquels les individus disent se conformer ou non. On distingue les «prescriptions » actives que sont les consignes données par le bailleur social après la réhabilitation thermique, les « incitations » relayées par les médias et les « informations » factuelles en lien avec la consommation d'énergie (factures, étiquetages énergétiques...).

5. Les éléments matériels appréhendés au travers de l'observation du système énergétique incorporé au logement (systèmes de chauffage, de ventilation et de production d'eau chaude sanitaire) et des équipements additionnels - les appareils acquis par le ménage (électroménager, équipements audiovisuels, électroniques, etc.)

Tableau 1. Une synthèse des principales approches de la théorie des pratiques

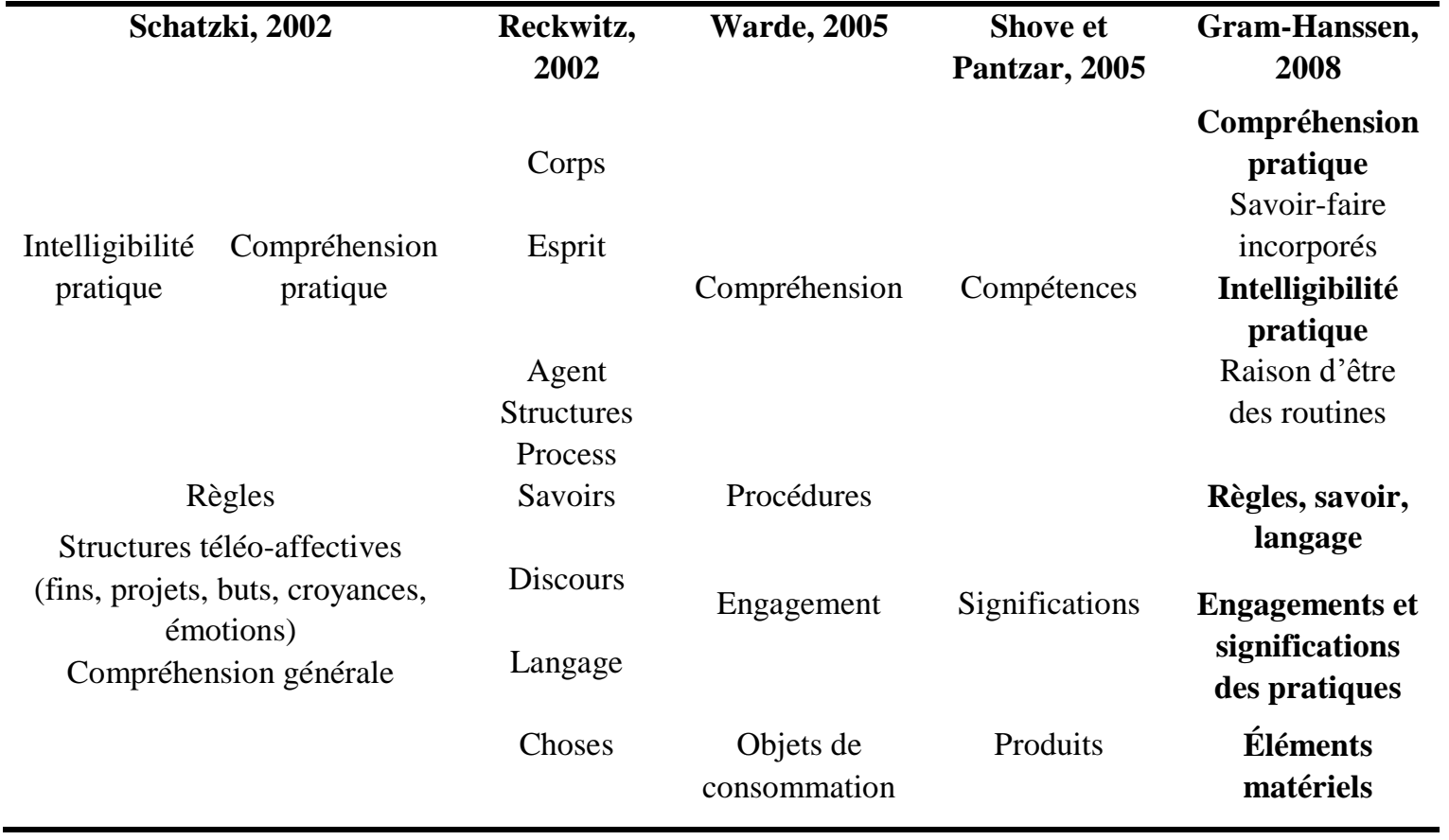

(source : Gram-Hanssen, 2008)

\section{Méthodologie de la recherche}

La collecte de données a été réalisée auprès de 40 individus (Annexe 1) dont la sélection est présentée dans l'encadré 2. L'étude comporte une phase d'observation et de recensement factuel des éléments du système matériel ; et une phase d'entretien orientée par le cadre de compréhension des pratiques (Gram-Hanssen, 2008). Ont été considérés, d'une part, l'acquisition d'appareils potentiellement consommateurs d'énergie à l'usage (équipements électroménagers et équipements audiovisuels, de 
loisirs et de communication), constituant de ce fait un premier «temps » de cette consommation, et d'autre part, les pratiques d'usage, moment de la consommation effective, en différenciant la gestion du système incorporé au logement et celle des équipements «meubles» acquis et implantés par le ménage. A ces deux types de pratiques - d'acquisition et d'usage -, les entretiens ont permis d'ajouter d'autres activités, non directement liées au système matériel, mais susceptibles de réduire la consommation d'énergie, même si celles-ci n'en constituent pas toujours la finalité première (par exemple, sortir de chez soi et pratiquer des activités non consommatrices d'énergie, inciter les autres à réduire leur consommation, etc.).

Encadré 2 : Echantillonnage et méthode d'analyse

L'étude a porté sur une population initiale de 200 locataires de logements réhabilités appartenant à quatre bailleurs sociaux de la Région Provence-Alpes-Côte d'Azur [Famille et Provence et Logirem à Vitrolles (13); Terre du Sud Habitat, à La Seyne sur Mer (83); Côte d'Azur Habitat à Nice (06)]. L'ensemble des quatre résidences concernées ainsi qu'au moins un appartement dans chacune d'elles ont été directement observés. Des entretiens ont été conduits avec les bailleurs sociaux et la maîtrise d'ouvrage déléguée pour comprendre les évolutions du système technique et les choix de réhabilitation thermique effectués.

Un échantillon de 40 individus a été ensuite extrait (à raison de 10 par bailleur) en faisant varier les principaux critères sociodémographiques - âge, genre, composition du ménage _ _ et dans les limitespour le niveau de formation, le revenu, et l'activité professionnelle, en respectant les caractéristiques de la population de locataires du logement socialeoncernée. Nous avons également : niveau de formation, revenus, activité professionnelle et en-veilléant à retenir des individus ayant, pour un tiers participé à une action particulière de sensibilisation (1, les deux autres tiers, -bien que conviés, n'y ayant ont pas participé), bien que conviés_-Introduire de la variance dans la participation au programme avait pour but d'était intéressant pour éviter de se-limiter à l'étude à d'une population particulière, plus responsabilisée, les travaux montrant que les individus les plus actifs dans la réduction énergétique sont aussi ceux qui sont les plus sensibilisés aux économies d'énergie (ADEME, 2016 ; Huzée et Cyssau, 2007). Au final, les individus présentent un grand nombre de caractéristiques communes : ils sont tous locataires, éligibles au logement social, situés dans le premier quintile de revenus et appartiennent aux catégories socio-professionnelles «employés », « ouvriers » ou « inactifs », avec un faible niveau de formation. En outre, ils n'ont aucun litige avec leur bailleur et occupent leur logement depuis au moins une année entière postérieurement à la réhabilitation. Les individus interrogés forment au final une population homogène dont l'hétérogénéité des pratiques de consommation énergétique est à comprendre à l'aune des engagements, des significations, des routines, de l'incorporation des normes, du suivi (ou non) des prescriptions externes en matière de réduction énergétique, et surtout du sens qu'ils donnent à ce qu'ils font (Annexe 1).

Les éléments observés dans le logement ainsi que les discours des répondants ont été codés en suivant la grille théorique de Gram-Hanssen (2008), faisant ainsi émerger des 
sous-dimensions illustratives. Pour chacune, le comportement observé et/ou déclaré du répondant a été codé comme favorable ou défavorable à la réduction de la consommation d'énergie. Les profils ont ensuite été agrégés, de proche en proche, selon leur densité (maximale vs. minimale) d'orientations favorables ou défavorables à la réduction énergétique. Nous avons d'abord séparé les profils aux pratiques majoritairement favorables $v s$. défavorables, puis affiné l'analyse de ces groupes en fonction des nuances qu'ils présentaient dans leurs pratiques : du côté favorable, les « volontaires » et les « responsables »; et du côté défavorable, les «empêchés » et les « réfractaires ». Ces profils définissent quatre «idéaux-types». Chaque individu est affecté à un et un seul profil en considérant le nombre de similitudes qu'il présente avec les caractéristiques les plus représentatives du profil. L'annexe 2 présente les quatre profils-types identifiés.

\section{Proposition d'une typologie d'individus et de leurs pratiques}

\section{favorables/défavorables à une réduction de la consommation d'énergie}

Le « volontaire » : sobre par conviction, allocentré et prosélyte

D’une manière générale, ce profil se caractérise par une capacité de compréhension des éléments techniques liés à ses équipements et une forte conscientisation de sa consommation, cherchant à communiquer aux autres son désir d'économie.

\section{Degré de compréhension pratique et d'appropriation des éléments incorporés}

Le système incorporé au logement ne semble pas lui poser de problème particulier comme l'illustre la compréhension, par Mélania, de la finalité de ses équipements : «Ils ont mis un double vitrage : la déperdition avec le chauffage est réglée. Maintenant on peut régler le chauffage ». Le fonctionnement de ce dernier ne crée aucune difficulté spécifique, pas plus que la gestion courante de ses appareils électriques domestiques.

\section{L'intelligibilité pratique et les raisons d'être des routines}

La consommation d'énergie du «volontaire» est fortement conscientisée dans les gestes quotidiens : "Eteindre les lumières en sortant d'une chambre », "attendre que le lave-linge soit plein pour éviter de faire des demi-charges », Jacqueline. A l'instar d'un couple étudié par Gram-Hanssen (2008) qui désire épargner et sait comment le faire, le «volontaire » se montre sobre par conviction, mais aussi par économie. «Les enfants ils disent : "Maman, il faut choisir ça, l'appareil il a ça, il a ça". Et nous on suit, on va acheter : moi je suis comme tout le monde », Nuria. Comme le relève à regret cette répondante, il bute sur certains compromis liés aux exigences de son entourage. 


\section{Les engagements et significations accordées aux pratiques}

Les aspirations du «volontaire » sont multiples et, de manière significative, très tournées vers l'extérieur (sortir de chez soi, prendre l'air, lire, jouer avec un enfant) : «J'ai du temps: je fais du vélo, je ne reste pas à la maison à regarder la télé », Laurence, tout en n'ayant pas pour finalité première la réduction de sa consommation d'énergie : «Je joue avec ma petite fille, je l'amène à un jardin, on regarde pas beaucoup la télé », Béatrice. Ce profil attache également beaucoup d'importance à la relation aux autres, aux activités pratiquées en commun : "Cet après-midi, on est allé à la bibliothèque avec les jeunes et il y a plein de jeux », Nuria. Il montre également un fort besoin d'ouverture et des valeurs morales et éthiques marquées (Innocent, FrançoisLecompte et Le Gall-Ely, 2016) : «Faut penser aussi à d'autres, parce que nous on a tout, le bon Dieu il nous a donné tout. Les pauvres, il y en a qui n'ont rien », Nuria. Alors que Gram-Hanssen (2008) passe sous silence la dimension sociale des pratiques, nos résultats montrent le rôle que ce profil joue sur son entourage, en étant fier de partager ses engagements à moins consommer : «Je parle avec les voisins parce qu'on est tous concernés, même si ça vient pas dans les conversations... On peut perdre ses habitudes », Carl. Le « volontaire » cherche de fait à être partie prenante des campagnes de réduction énergétique en relayant les informations autour de lui. Il manifeste une forte dimension «réalisation de soi » (Innocent, François-Lecompte et Le Gall-Ely, 2016) qui s'exprime au travers ses talents de communication et sa volonté d'inciter les autres au changement : "L'union fait la force. On peut éclaircir, on peut montrer des flyers, montrer aux gens que ça marche, faire venir des amis à la maison. Regardez! Nous on ne consomme pas, on paye moins cher que certains autres », Nuria.

\section{Les règles, prescriptions et influences}

Le «volontaire », qui s'alimente des sources d'informations environnantes, tend à s'y conformer et à les mettre à profit : «On trouve plein de chose dans les associations, on entend plein de choses, on écoute et à chaque fois on passe le message à nos enfants, aux gens qu'on connaît et tout ça », Béatrice. Chez ce profil largement préoccupé par les niveaux de consommation d'énergie des appareils acquis, il n'y a pas d'aspiration particulière à un niveau de confort plus élevé : «Maintenant lorsque vous achetez un appareil ménager c'est écrit ce qu'il va dépenser. Vous allez prendre un A+ou A, vous 
n'allez pas prendre $B, C, D \gg$, Jacqueline. Il manifeste simplement une attention aux prescriptions en faveur d'équipements les moins énergivores possibles.

\section{Les éléments matériels du système énergétique incorporé et acquis}

Le «volontaire » se satisfait en général d'un niveau d'équipement standard : « $J^{\prime} a i$ aménagé par rapport à nous. J'aime bien cuisiner : il y a un peu de matériel», Murielle, même si, sous la pression de l'entourage et notamment des enfants, il peut avoir tendance à céder à multiplier ou renouveler ses équipements (téléphonie par exemple).

Le « responsable » : efficace sans perte de confort et sans changer le monde Son approche pragmatique fait de ce profil le champion économique de la «chasse au gaspi » en dépit d'une faible conscientisation des consommations associées aux équipements acquis du fait qu'il ne souhaite pas se priver d'un certain confort.

\section{Degré de compréhension pratique et d'appropriation des éléments incorporés}

Ce profil est globalement attentif aux performances des matériels et à la gestion du système incorporé dont il s'applique à comprendre et s'approprier l'usage, comme Bernard, très attentif aux explications de l'animateur de la campagne de sensibilisation : «Le monsieur de l'énergie m'a dit que c'était un thermostat et quand il fait froid ça s'allume et quand il fait un peu chaud ça baisse, c'est automatique ».

\section{L'intelligibilité pratique et les raisons d'être des routines}

Le « responsable » est conscient de sa consommation dans ses moindres routines : «Je ne laisse jamais les appareils en veille », Maya; "Je me suis habitué à éteindre tout, même si je paye pareil », Bernard. Si, incidemment, son niveau de consommation lui est rappelé, par les factures notamment, il vit sa situation comme le résultat d'un contexte ou de limites sur lesquels il ne peut agir : «Avant, il y avait la campagne, ils pouvaient aller jouer dehors. Je vais leur dire quoi? Allez jouer dans le parking ? Il faut bien les occuper, on a peur quand ils sortent», Paméla. L’intelligibilité des pratiques bute de fait contre des besoins perçus comme fondamentaux et irréductibles : «Chez moi je ne peux pas faire grand-chose puisqu'il y a actuellement tout d'éteint sauf le téléphone. Ce n'est pas à moi qu'il faut dire ça, c'est aux autres qui consomment beaucoup », Bernard. Ce qui dépasse le cadre strict de son action débouche ainsi sur un report de responsabilité sur d'autres acteurs.

\section{Les engagements et significations accordées aux pratiques}


Le «responsable» est en quelque sorte le champion de l'efficacité, tant lors de l'acquisition d'équipements supplémentaires - «Je préfère qu'elle soit en classe A et qu'elle consomme moins d'électricité et qu'elle consomme moins d'eau », Madona que dans la chasse au gaspillage, par exemple avec un équipement quasi-systématique en ampoules basse consommation ou l'extinction systématique de ce qui consomme «pour rien » et ne contribue pas immédiatement au confort. L'intérêt financier de ses comportements, toutefois, est patent: «Le but c'est économiser l'énergie et le portefeuille à la fin de l'année », Pierre. Porté par sa conception enthousiaste d'un comportement efficace, le «responsable» ne semble guère percevoir la possible dissonance qui existe entre ses micro-gestes économes et son niveau d'équipement, souvent élevé, et donc fortement consommateur d'énergie.

\section{Les règles, prescriptions et influences}

Sensible à la réduction de la consommation d'énergie, le « responsable » désire bien faire en suivant les prescriptions ou les informations fournies. Cette orientation fait que le «responsable» est un participant actif à la campagne de sensibilisation, mais seulement sur les comportements de son entourage direct, quitte à s'y substituer : « $L a$ veille des téléviseurs, il parait qu'il faut les éteindre. Mon mari, il dit que c'est ridicule parce qu'il est obligé de la rebrancher. Je l'éteins derrière lui », Sophie. Au final, le « responsable» désire bien faire : «Pour avoir un groupe, il faudrait déjà avoir des gens motivés : chez nous ils ne sont pas motivés. Je me recentre sur ma famille », Josette, mais contrairement au «volontaire », il s'en tient à agir sur ce qu'il maîtrise, à son niveau, sans la dimension prosélyte qui caractérise le profil précédent.

\section{Les éléments matériels du système énergétique incorporé et acquis}

Les résultats montrent que la volonté du «responsable» de réduire sa consommation trouve sa limite dans celle de ne pas se priver : «On adapte tout pour se sentir bien. Le grand écran [pour la télévision] on s'est fait plaisir... Il est en 3D, il fait 140 de dimension. Les enfants sont autonomes : ils ont la télé, la chaîne hi-fi, la console, la tablette, la totale », Paméla. Ces besoins se traduisent de fait par le nombre et les caractéristiques des appareils possédés (audio-visuel, informatique, communication) qui engendrent une forte croissance de la consommation d'énergie spécifique non prise en compte dans l'approche de Gram-Hanssen (2008). 


\section{L'« empêché » : frugal malgré lui}

Dans les profils orientés plutôt défavorablement, 1' « empêché » est caractérisé non seulement par des revenus faibles, une situation sociale difficile ou précaire (telle que famille monoparentale, faibles revenus, handicap, activité réduite ou chômage), mais aussi par une difficulté à peser sur le cours des choses. Se percevant vulnérable, il est méfiant et tend à se replier sur lui-même.

\section{Degré de compréhension pratique et d'appropriation des éléments incorporés}

Sur le registre de la performance des systèmes incorporés au logement, l' « empêché » ressent particulièrement l'existence de défauts et dysfonctionnements, générateurs d'inconfort et consommateurs d'énergie. La gestion de la température est problématique aussi, car la compréhension de certains systèmes n'est pas assurée ou incomplète : «Je ne sais pas si c'est utile les robinets thermostatiques. Moi, quand il fait froid, je les mets au maximum », Francine. Des gestes maladroits, voire contre-performants, peuvent même ainsi apparaître - ouvrir les fenêtres plutôt que réguler la température avec les robinets thermostatiques - et ceci d'autant plus si les équipements incorporés présentent des dysfonctionnements. En cela, il fait écho à l'un des couples interrogés par GramHanssen (2008), étonné, par méconnaissance des dispositifs de régulation, de ses faibles performances énergétiques en dépit de la température basse du logement.

\section{L'intelligibilité pratique et les raisons d'être des routines}

Tout en étant conscient de l'intérêt de tenir compte un jour de l'efficacité énergétique ${ }^{7}$, l'«empêché » ne parvient pas à y investir. En cela, il confirme le constat critique de Moloney et Strengers (2014) pour qui la responsabilité des gestes écologiques est imputée aux individus, dont une partie d'entre eux ne peut pas financièrement les assumer. L' « empêché » ne voit donc pas comment faire plus ou autrement que ce qu'il fait déjà : «Pour moi, pour l'instant, c'est machinal. Ce que je fais je ne pourrais pas l'enlever, on ne peut pas enlever ses habitudes comme ça, malgré que l'on soit dans un quartier spécial », Françoise. De fait, la référence aux routines se manifeste sous l'angle d'une continuité existentielle, structurante, et donc difficilement modifiable.

\section{Les engagements et significations accordées aux pratiques}

\footnotetext{
${ }^{7}$ Sur la question des incitations à la réduction de la consommation d'énergie, l'étude ADEME (2016) souligne que $53 \%$ des répondants sont d'abord guidés par des considérations économiques, $29 \%$ citant l'augmentation de leur facture énergétique et $24 \%$ le fait que leur situation financière l'impose. De même, une étude l'enquête Environnement Opinion Way 2015 pour l'ADEME indique qu'être locataire est le premier frein à la réalisation de travaux de rénovation énergétique.
} 
En dépit de ses difficultés à s'extraire de sa situation, l'« empêché » demeure ouvert à de possibles changements : «Oui, c'est possible de réduire la consommation d'énergie de moitié mais il faut trouver une astuce et quand on a cinq enfants sur le chemin ce n'est pas simple », Moktar. Si Aline tente de faire agir ses proches : «Au quotidien, je le dis aussi à ma fille pour essayer de la sensibiliser un peu. Éteins l'eau, éteins la lumière quand tu sors de ta chambre. Dès qu'il y a un truc, éteins-le», son influence sur les autres n'est pas absente, mais reste d'ampleur modeste.

\section{Les règles, prescriptions et influences}

En raison de ses contraintes, l'«empêché » est sensible à l'information mais son attention se porte davantage sur le montant de sa facture d'énergie que sur le niveau réel de sa consommation. Il existe même parfois une confusion entre facturation et quantité consommée, par exemple lorsque le bailleur social lui reverse un trop perçu (en fonction de la réalité de ses charges), interprété à tort comme une baisse de sa consommation. Malgré une méfiance, voire une certaine défiance aux autres, l'«empêché » peut manifester une attente par rapport à l'apport potentiel de tiers de confiance identifiés : «Je ne vois pas comment je pourrai réduire ma facture, je demanderai conseil. Je m'adresserais à EDF, c'est les seuls qui pourront nous donner des conseils à ce niveaulà », Francine. De fait, sa situation sociale d'isolement nuit à la réceptivité et à l'acceptation des prescriptions qui se heurtent souvent à son sentiment d'impuissance.

\section{Les éléments matériels du système énergétique incorporé et acquis}

Se restreignant par nécessité absolue du fait de sa situation économique, l' «empêché » dispose d'un niveau d'équipement personnel souvent basique, le coût d'appareils performants étant souvent généralement hors de portée financière : "J'ai une seule télé, c'est un écran d'il y a très longtemps : c'est encore un tube cathodique », Aline. Au final, la question qui se pose à propos de ce profil est la direction vers laquelle il pourrait «migrer » en cas de desserrement de ses contraintes.

Le « réfractaire » : quand réduire, c’est régresser

A l'extrémité du continuum d'orientations défavorables, le « réfractaire » refuse toute contrainte énergétique, manifestant un désintérêt pour une démarche de réduction, que celle-ci soit perçue comme une régression ou comme une peur de perdre sa liberté.

\section{Degré de compréhension pratique et d'appropriation des éléments incorporés}


Ce qui le caractérise de manière évidente, c'est son incompréhension délibérée, son désintérêt pour une meilleure maîtrise de l'usage de l'énergie, ou encore ses approximations ou interprétations erronées des systèmes techniques, qu'il s'agisse des équipements acquis comme l'illustre Fabienne : "J'ai tous les appareils qui restent en veille, la chaîne hi-fi, tout ça, mais ça consomme pas beaucoup », mais aussi du système de chauffage incorporé.

\section{L'intelligibilité pratique et les raisons d'être des routines}

Globalement, ses pratiques ne sont pas porteuses de réduction ou de maîtrise de sa consommation, mais empreintes de fatalisme, avec l'idée qu'on ne peut rien y changer (Moloney et Strengers, 2014) : «C'est comme la taxe d'habitation, on n'a pas le choix, on la paye », René. Pour la plupart, les routines quotidiennes, bien qu'énergivores, sont justifiées par le désir d'éviter des efforts cognitifs qui viseraient à changer l'existant : «La télé est en veille $24 \mathrm{~h} / 24$, la télé...c c'est une question de commodité et en fait je ne me suis jamais posé la question », Lucette. De même, le critère de consommation énergétique n'est jamais pris en compte lors de l'achat d'appareils additionnels.

\section{Les engagements et significations accordées aux pratiques}

Le «réfractaire»se caractérise par un refus marqué de toute contrainte énergétique. Bien qu'il affirme ne pas gaspiller, on perçoit dans son orientation une forme d'inertie, voire de résistance. Toutes les pratiques sont donc considérées comme irréductibles : on ne peut pas faire moins, on ne peut pas faire mieux. Pour les plus âgés comme Daniel (80 ans), c'est la crainte de régresser qui domine, par référence au passé, à ce qui a pu être acquis ou conquis en termes de niveau de vie et de confort : «On revient à la lampe à huile de mon grand-père...A un certain âge j'ai connu la guerre, les restrictions, le manque de ceci, le manque de cela. En faire plus, non, c'est pas possible ». Pour les plus jeunes, c'est le sentiment de perte de liberté ou de diminution du confort qui est patent. Quel que soit l'âge, on constate également un refus d'anticiper l'avenir comme le montre Mahmoud: «On n'a qu'une seule vie, pourquoi on va se priver de plein de choses alors qu'on peut vivre normalement? », soulignant un ancrage hédoniste dans l'ici et maintenant par défaut de visibilité du lendemain.

\section{Les règles, prescriptions et influences}

Le «réfractaire» fait parfois référence à des tensions face auxquelles la pression à la réduction de consommation d'énergie est ressentie comme inadmissible. En découle un 
rejet de la responsabilité que le discours écologique cherche à faire porter aux individus (Moloney et Strengers, 2014), responsabilité que le « réfractaire » reporte sur d'autres : «C'est plus les grandes sociétés qui devraient faire attention. Ceux qui brûlent, les usines, c'est plus à eux de défendre la planète », Mickaël. De fait, le « réfractaire » est peu perméable à l'influence, aux prescriptions ou aux informations fournies sur la consommation : "C'est pas facile la différence entre le double A et le triple A. Même les vendeurs ils ne savent pas. Tout ça c'est de la rigolade», Rose. En revanche, à l'instar de Mila qui indique : "Ça serait bien d'encourager de cette manière : s'il y a un bonus, je pense qu'il y aura plus de personnes qui feront attention », il se déclare sensible aux récompenses économiques comme possibles leviers de changement, une suggestion déjà formulée par Bertoldi, Rezessy et Oikonomou (2013).

\section{Les éléments matériels du système énergétique incorporé et acquis}

S'agissant de son niveau personnel d'équipement, le « réfractaire » n'est pas caractérisé par une diversité ou un nombre d'appareils particuliers, sa limite étant surtout liée à sa capacité financière.

En résumé, le cadre d'analyse fourni par la théorie des pratiques permet de mettre en relief les dimensions et composantes les plus caractéristiques de chaque profil, facilitant ainsi l'identification des leviers de la réduction de la consommation d'énergie. Parmi les individus orientés favorablement - volontaires et responsables - l'analyse montre que la dimension la plus contrastée est l'intelligibilité pratique et les raisons d'être des routines incorporées. Ainsi, là où le volontaire cherche à faire agir les autres, le responsable privilégie le résultat immédiat et tend à se substituer à eux pour agir à leur place. Parmi les individus orientés défavorablement - réfractaires et empêchés -, les résultats montrent que la dimension la plus contrastée porte sur les engagements et significations. Chez le réfractaire, les pratiques sont choisies tandis que pour l'empêché elles sont essentiellement contraintes. Le second caractère distinctif relève du degré de compréhension pratique, l'empêché cherchant à gérer au mieux sa consommation que le réfractaire qui considère l'existant comme inamovible. 


\section{Discussion et implications pour l'action}

Les résultats ci-dessus, appuyés sur la théorie des pratiques, soulignent l'enchevêtrement de facteurs générateurs de consommation d'énergie et de freins à sa réduction. Toutefois, chacun des profils identifiés présente des leviers d'action spécifiques que nous détaillons à la suite (Tableau 2).

\section{Tableau 2 : Synthèse des profils, objectifs et modalités d'action pour favoriser la réduction de la consommation d'énergie}

\begin{tabular}{|c|c|c|c|}
\hline «Profil » & $\begin{array}{l}\text { Caractéristiques principales } \\
\text { du profil }\end{array}$ & Principaux objectifs d'action & Modalités d'action spécifiques \\
\hline Volontaire & $\begin{array}{l}\text { Être partie prenante de sa } \\
\text { maîtrise énergétique }\end{array}$ & $\begin{array}{l}\text { L'inciter à exprimer ses convictions, } \\
\text { l'inciter à se comporter comme un } \\
\text { leader d'opinion }\end{array}$ & $\begin{array}{l}\text { Soutenir sa propension à agir sur les autres } \\
\text { Lui donner un rôle de block-leader dans } \\
\text { l'immeuble et les associations de quartiers }\end{array}$ \\
\hline Responsable & $\begin{array}{l}\text { Etre efficace mais sans } \\
\text { remise en cause de son } \\
\quad \text { « confort }\end{array}$ & $\begin{array}{l}\text { Consolider sa propension à l'efficacité } \\
\text { Dissocier la réduction énergétique de } \\
\text { l'idée de privation }\end{array}$ & $\begin{array}{l}\text { Accroître son accessibilité aux équipements } \\
\text { efficaces et améliorer l'efficacité de gestion de } \\
\text { ses équipements } \\
\text { Promouvoir des alternatives sobres (pratiquer } \\
\text { un sport, participer à des activités collectives) }\end{array}$ \\
\hline Empêché & $\begin{array}{c}\text { Etre dans l'incapacité } \\
\text { économique à faire plus ou } \\
\text { mieux en dépit d'une possible } \\
\text { volonté de changement }\end{array}$ & Rompre l'isolement & $\begin{array}{l}\text { Multiplier les caractéristiques efficaces des } \\
\text { équipements à prix inchangé } \\
\text { L’inciter à partager des équipements } \\
\text { Favoriser le « parrainage » entre voisins (par le } \\
\text { « volontaire ») }\end{array}$ \\
\hline \multirow[t]{2}{*}{ Réfractaire } & $\begin{array}{c}\text { Etre dans la peur de la } \\
\text { réduction de consommation } \\
\text { assimilée à une régression... }\end{array}$ & $\begin{array}{c}\text { Montrer l'intérêt financier de l'efficacité } \\
\text { d'usage sans incidence sur le service } \\
\text { rendu }\end{array}$ & $\begin{array}{l}\text { Donner des exemples concrets avec chiffrage } \\
\text { des gains }\end{array}$ \\
\hline & ...ou à une entrave & $\begin{array}{c}\text { Assurer l'efficacité des équipements } \\
\text { hors de l'utilisateur } \\
\text { Lui donner envie d'imiter les autres }\end{array}$ & $\begin{array}{l}\text { Systématiser l'incorporation des caractéristiques } \\
\text { efficaces dans les appareils (coupure de veille } \\
\text { des appareils) } \\
\text { Promouvoir des modèles socialement valorisants, } \\
\text { corroborés par des témoignages de proximité }\end{array}$ \\
\hline
\end{tabular}

\section{Donner au « volontaire » le rôle de leader d'opinion dans son entourage}

Les individus se rattachant au profil «volontaire » et dans une certaine mesure, au profil « responsable », manifestent, au-delà de leur propre pratique, une satisfaction et même une fierté à s'engager dans la maîtrise de leur consommation, dans laquelle ils trouvent une valeur morale et de réalisation (Innocent, François-Lecompte et Le Gall-Ely, 2016 ; Özçağlar-Toulouse, 2009). Cette implication déborde du cadre personnel pour souligner la valeur sociale de ces comportements (Bertrandias, 2012). Il est donc important 
d'inciter ces individus à exprimer leurs convictions, à se comporter comme des leaders d'opinion car, se voyant attribuer une conscience écologique par les personnes de leur entourage, ils sont susceptibles de les aider à faire évoluer leurs pratiques. Le «volontaire » pourrait alors se voir confier des actions de sensibilisation lui conférant un rôle d'entraînement ou de block-leader (Hopper et Nielsen, 1991). Comme le montrent ces auteurs dans le domaine du tri/recyclage, l'effet d'imitation de ces volontaires modifie les comportements de leurs voisins sans qu'il y ait besoin d'agir sur leurs attitudes. A la différence des actions de sensibilisation de type top-down (Moloney et Strengers, 2014), les relations sociales de voisinage créent une «accessibilité spatiale » qui produit et pérennise de tels changements (Bertrandias, 2012). En outre, la proximité et la similitude de situation que constitue «l'accessibilité sociale» (Bertrandias, 2012) favorisent l'écoute et l'acceptation par mimétisme, alors que des organismes extérieurs sont parfois perçus comme lointains, sinon intéressés à la promotion d'un discours écologique responsabilisant (Moloney et Strengers, 2014). En complément, un «parrainage » de voisinage permettrait de créer une relation de confiance entre le « volontaire » et le parrainé, inscrivant l'action dans la durée, la quotidienneté et l'espoir de voir partager l'action par plus d'individus. L'enrôlement des «volontaires » pourrait se faire par le biais des personnels accompagnant la mise en place des programmes, à la fois pour détecter ces profils et leur fournir un local et des documents d'information à diffuser et discuter lors de ces rencontres.

\section{Aider le « responsable » à passer de l'efficacité énergétique à la sobriété}

Recherchant activement de l'information et pouvant consentir à des efforts de gestion afin de minimiser sa consommation d'énergie pour un service rendu identique (efficacité), le « responsable »n'accepte pas pour autant l'idée d'une privation associée à la sobriété. S'il accepte de réduire la consommation de ses équipements (et par là son impact financier), c'est donc en adoptant un comportement d' «altruiste » calculateur (Hopper et Nielsen, 1991) qui s'insère facilement dans son mode de vie et n'implique aucune renonciation à ses pratiques. Plusieurs recommandations s'imposent. En premier lieu, il est essentiel d'entretenir sa propension à l'efficacité en lui conseillant les équipements les plus performants et en lui fournissant des préconisations de gestion de ses appareils. En second lieu, il convient aussi de le sensibiliser à l'effet rebond auquel 
il ne lie pas toujours nécessairement la possession d'équipements supplémentaires. Ainsi, il convient de l'informer que sa consommation n'est pas seulement liée à la gestion de ses appareils, mais aussi à son niveau d'équipement. Cela suppose d'agir sur la conscientisation des pratiques gourmandes en équipements nouveaux ou supplémentaires et sur la manière dont celles-ci s'enracinent dans la vie des individus (Shove, 2010). Bien que Shove souligne la difficulté du travail de déconstruction de l'existant, pourtant essentiel à un véritable changement, des pistes peuvent être trouvées en incitant ces individus à adopter des activités substitutives (Spurling et alii, 2013), notamment celles qui, comme le sport, les activités sociales, la fréquentation d'une bibliothèque ou d'un espace collectif dans le quartier, sont sobres en énergie. Cette réorientation des pratiques en dehors du logement, espace de forte consommation pourrait avoir prise sur ce profil sensible aux discours environnementaux. Par ailleurs, une autre piste est à retenir du côté des fabricants/distributeurs qui consisterait d'une part à valoriser, dans les publicités des modèles sobres que le «responsable » aurait envie d'imiter, d'autre part à ré-élaborer les composants matériels de la pratique en développant l'efficacité énergétique et l'éco-design des produits (Spurling et alii, 2013).

\section{Rompre l'isolement et desserrer les contraintes de l'«empêché »}

Ce profil, particulièrement vulnérable et contraint, présente néanmoins une sensibilité environnementale qui mérite d'être prise en compte. C'est en premier lieu sur l'efficacité énergétique que les efforts doivent porter, plutôt que sur la sobriété à laquelle ces individus sont contraints. Aussi, maintenir un niveau de qualité irréprochable des systèmes incorporés (pour le chauffage, la ventilation, l'eau chaude sanitaire) et leur fournir des modes d'emploi facilement compréhensibles sur la manière de s'en servir sont deux priorités pour éviter qu'ils n'adoptent des gestes contreperformants. En second lieu, dans le sillage des initiatives collaboratives, le partage, la mutualisation ou le prêt d'appareils entre voisins pourraient venir pallier la faiblesse de leurs équipements, sans surcoût financier et sans impact écologique supplémentaire. Ainsi, Borel, Roux et Demailly (2016) soulignent les motivations sociales à échanger des objets dans un réseau de prêt entre voisins à Toulouse où l'interconnaissance est forte. Par ailleurs, l'«empêché » exprime généralement le besoin d'échapper à son contexte socio-économique fait d'isolement, de précarité, d'insécurité, de vulnérabilité, 
de manque d'information. Stimuler des orientations sociales plus positives - discuter avec d'autres, participer à un projet collectif, et échanger des «tuyaux » sur de bonnes pratiques à la fois économiques et écologiques - pourrait être une piste prometteuse. Un tiers de confiance tel un proche, une personne connue (voisin, gardien...) pourrait servir de médiateur de proximité, rôle qui pourrait également être confié au «volontaire » dont le caractère prosélyte et le rôle de block-leader a été évoqué plus haut. Une telle formule de parrainage pourrait donc être une première voie de passage, sécurisée et sécurisante, pour des individus souvent repliés sur eux-mêmes et peu confiants dans leurs moyens de progresser.

\section{Libérer le « réfractaire » du sentiment de régression ou d'entrave}

Le « réfractaire » est celui qui oppose les plus fortes résistances au changement de comportement énergétique. La perspective d'une réduction (à la fois des équipements et de la consommation induite par leur utilisation) est associée à une sobriété contrainte qui peut être ressentie comme une atteinte à sa liberté ou à son plaisir (Innocent, François-Lecompte et Le Gall-Ely, 2016). Ces peurs sont alors une façon de légitimer son blocage à réduire sa consommation. De fait, les risques de résistance à des formes de communication trop injonctives appellent des solutions plus subtiles, basées sur l'architecture de choix. Celle-ci peut consister, en phase d'acquisition, à le diriger vers des équipements dotés d'un haut niveau d'efficacité qui pourraient ainsi réduire sa consommation énergétique sans altérer le service et sans éveiller un sentiment de régression ou d'entrave. En outre, ces équipements pourraient être dotés de fonctionnalités favorisant une utilisation elle-même efficace, par exemple un dispositif par défaut d'interruption automatique de la veille, incluant une fonction de réactivation aussi simple d'usage qu'une télécommande. Si ces préconisations ne sont pas propres au « réfractaire », elles doivent être généralisées en raison même de l'existence de ce profil, peu enclin à suivre des directives ou à les appliquer spontanément. En second lieu, mettre en avant les gains économiques obtenus sans incidence sur le service attendu serait de nature à valoriser l'intérêt de l'efficacité énergétique en phase d'usage. Il serait donc profitable de fournir au réfractaire, très attentif aux charges occasionnées par sa consommation énergétique, des encouragements sur les économies réalisées au fil du temps qui, actuellement, ne sont pas directement visibles dans ses factures. La 
généralisation des nouveaux «compteurs intelligents» pourrait jouer ce rôle de rétroaction en lui donnant le sentiment d'être maître de sa consommation et de ses dépenses. Plus encore, le «réfractaire» se déclarant réceptif à l'idée d'une récompense ou d'un « bonus », un programme d'incitations financières ex ante liées aux économies réalisées, couplé à un système de suivi en temps réel de leur consommation pourrait se révéler intéressant (Bertoldi, Rezessy et Oikonomou, 2013 ; Zhao et alii, 2012). Cette recommandation concerne en particulier les bailleurs sociaux et l'information qu'ils pourraient leur fournir, à la fois sur leurs gains de consommation et sur les économies réalisées. En troisième lieu, ces mêmes acteurs pourraient publier des chiffrages de consommation comparatifs dont l'efficacité a été démontrée (Allcott, 2011). En effet, la théorie de l'engagement appliquée au domaine de l'énergie montre que les individus auxquels on demande un geste - par exemple réduire l'usage d'une climatisation $-\mathrm{s}$ 'y tiennent quand cet engagement est public et l'oublient quand il est privé (Pallack et Cummings, 1976). Au final, des systèmes d'incitation douce pariant sur des effets d'imitation pour orienter les comportements dans le sens souhaité (Thaler et Sunstein, 2008) pourraient avoir prise sur les « réfractaires » sans les priver de liberté. Au-delà de chaque profil, une recommandation d'ensemble concerne la tarification actuelle de l'énergie. Il conviendrait en effet de remplacer le système dégressif actuel (le prix de revient du $\mathrm{kWh}$ diminue avec la consommation) par une tarification progressive de l'énergie (le prix du kWh consommé augmente avec la consommation), qui se révèlerait plus cohérente pour dissuader toute tendance à épuiser la ressource.

Au-delà, notre recherche présente un intérêt plus général pour les acteurs de la communication publique environnementale (Ministère de la Transition écologique et solidaire, ADEME ou fournisseurs d'énergie). En effet, la réduction de la consommation d'énergie peut résulter de l'efficacité et/ou de la sobriété : il importe donc de considérer le point de vue du consommateur différemment de celui du fournisseur d'énergie. En effet, si pour ce dernier l'unité de mesure est la quantité d'énergie consommée (quel que soit le service), pour le premier, c'est le volume ou la qualité de service obtenu qui se révèle essentiel. Il est donc nécessaire que la communication publique insiste sur la maximisation de l'expérience de service de l'utilisateur plutôt que de lui faire craindre sa dégradation. La communication visant la sobriété doit également tenir compte des profils. Elle s'adresse prioritairement au 
« responsable » et au «volontaire», et non au «réfractaire» pour qui les discours de réduction risquent de susciter de la résistance et appellent d'autres actions, par exemple des incitations économiques, moins problématiques sur ce point.

\section{Conclusion}

Les individus, au quotidien, consomment de l'énergie dans leur cadre résidentiel, c'està-dire dans ce qui est à proprement parler le «foyer » de leur existence, objet de toutes les attentions et forme d'extension de soi. Il apparaît toutefois que la consommation d'énergie n'est ni une fin, ni une pratique en soi, mais « un moment» au sein d'autres pratiques (Warde, 2005). Elle est la contrepartie nécessaire d'autres activités inductrices de consommation énergétique pour un service attendu. L'énergie a également comme particularité de ne pouvoir être utilisée directement: sa consommation est nécessairement liée à celle d'équipements spécifiques à chaque service. Prenant appui sur la théorie des pratiques et son application au contexte de la consommation d'énergie (Gram-Hanssen, 2008), nous avons montré, à l'instar de Spurling et alii (2013), que les pratiques ne sont pas le simple résultat d'un choix individuel, ni d'une appréciation des simples coûts et bénéfices liés à la maîtrise des consommations énergétiques (Innocent, François-Lecompte et Le Gall-Ely, 2016), mais qu'elles sont enchâssées socialement et matériellement. En cela, la théorie des pratiques propose un éclairage systémique des comportements qui évite deux grands écueils (Geels et alii, 2015) : soit considérer, dans une perspective «révolutionnaire », que la sobriété ne peut être atteinte qu'en suivant les préceptes de la décroissance, sans considérer le caractère socialement marqué et souvent élitiste de ces mouvements ; soit penser, dans une perspective «réformiste », qu'il suffit de peser sur les attitudes ou les choix pour obtenir des effets durables sur les comportements (Allcott, 2011). Ces deux approches relèvent de positions idéologiques opposées mais se rejoignent sur le principe d'une (ir)rationalité des décisions prises par les individus. A contrario, notre approche considère que l'individu est d'abord pris dans des routines, enserré dans un système de normes sociales et contraint par un environnement socio-technique particulier. Nous considérons, à l'instar de GramHanssen (2008) que la théorie des pratiques est pertinente et utile pour l'analyse de ces comportements. Notre étude enrichit la théorie à la fois dans son application et dans son exploitation: en intégrant toutes les activités susceptibles de peser sur leur 
consommation, notamment l'acquisition d'équipements électriques supplémentaires audelà du seul système incorporé au logement; en proposant une typologie utile à la formulation de recommandations différenciées pour chaque profil selon le caractère favorable ou défavorable de leurs activités/comportements. Nos résultats comportent toutefois certaines limites, portant tout d'abord sur le contexte d'observation. Il est ainsi possible que d'autres environnements puissent faire émerger de nouveaux profils, ou d'autres nuances dans les pratiques. Par exemple, il n'est pas sûr que les « volontaires » observés ici soient similaires à ceux qui pourraient assumer un rôle de leader d'opinion dans un quartier résidentiel avec des habitations individuelles. L'analyse pourrait également être poursuivie dans le sens de l'approfondissement du rôle des sources d'influence, informatives ou prescriptives, et des phénomènes d'acceptation, de rejet ou de résistance qu'elles peuvent engendrer. En effet, les techniques de nudge, a priori jugées peu intrusives, ne seront efficaces que si (ou tant que) les individus ne les perçoivent pas comme des tentatives de manipulation de leurs comportements. Enfin, des études quantitatives pourraient utilement compléter cette première approche, en incluant notamment la mesure de l'idéologie politique. En effet, celle-ci semble entraîner une réception différente des nudges selon la sensibilité idéologique des individus (Costa et Kahn, 2010), appelant ainsi à leur usage différencié selon les profils. Finalement, il apparaît aussi que les individus sont soumis à des injonctions paradoxales - consommer vs. économiser, optimiser leurs usages vs. réduire leurs équipements. Il serait donc utile d'analyser, dans une perspective systémique, la manière dont différents types d'acteurs - économiques, institutionnels et pro-environnementaux - s'empare de la question énergétique et pèsent, parfois de manière contradictoire, sur leurs comportements.

\section{Références}

ADEME (2016), Changer les comportements, faire évoluer les pratiques sociales vers plus de durabilité. L'apport des sciences humaines et sociales pour comprendre et agir, Rapport d'étude.

Allcott H. (2011), Social norms and energy conservation, Journal of Public Economics, 95, 9-10, 1082-1095. 
Bertoldi P., Rezessy S. et Oikonomou V. (2013), Rewarding energy savings rather than energy efficiency : Exploring the concept of a feed-in tariff for energy savings, Energy Policy, 56, 526-535.

Bertrandias L. (2012), Influences informationnelle et normative sur les comportements du consommateur, Mémoire en vue de l'obtention de l'Habilitation à Diriger des Recherches en Sciences de Gestion, Université Toulouse Capitole.

Borel S., Roux D. et Demailly D. (2016), La place des enjeux sociaux et environnementaux dans la consommation collaborative : le point de vue des usagers, PICO Working paper, Paris, IDDRI.

Bourdieu P. (1980), Le sens pratique, Paris, Editions de Minuit.

Bourg D., Dartiguepeyrou C., Gervais C. et Perrin O. (2016), Les nouveaux modes de vie durables. S'engager autrement, Lormont, Edition le Bord de l'Eau.

Brisepierre G. (2011), Les conditions sociales et organisationnelles du changement des pratiques de consommation d'énergie dans 1'habitat collectif, Thèse de sociologie, Université Paris Descartes.

Costa D. et Kahn M. (2010), Energy conservation nudges and environmentalist ideology: evidence from a randomized residential electricity field experiment. NBER Working Paper, $\mathrm{n}^{\circ} 15939$.

Dubuisson-Quellier S. et Plessz M. (2013), La théorie des pratiques Quels apports pour l'étude sociologique de la consommation ?, Sociologie, 4, 4.

https://sociologie.revues.org/2030, consulté le 3/12/2016.

Dujin A., Maresca B. et Vedie M. (2012), Changer les comportements. L'incitation comportementale dans les politiques de maîtrise de l'énergie en France, Paris, Cahiers de Recherche du CREDOC, $\mathrm{n}^{\circ} 295$.

Geels F.W., McMeekin A., Mylan J., Southerton D. (2015), A critical appraisal of Sustainable Consumption and Production research: The reformist, revolutionary and reconfiguration positions, Global Environmental Change, 34, 1-12

Giddens A. (1984), The constitution of society: Outline of the theory of structuration, Cambridge, Polity Press.

Gram-Hanssen K. (2008), Heat comfort and practice theory. Understanding everyday routines of energy consumption, Proceedings of the SCORE Conference, Brussels, mars, 53-72.

Hopper J.R. et Nielsen J.M. (1991), Recycling as altruistic behavior: normative and behavioral strategies to expand participation in a community recycling program, Environment and Behavior, 23, 2, 195-220.

Huzée M.-H. et Cyssau R. (2007), Maîtrise de la demande d'énergie par les services d'individualisation du chauffage, Rapport de recherche pour le compte de l'ADEME. 
Innocent M., François-Lecompte A. et Le Gall-Ely M. (2016), La valeur associée à la maîtrise de la consommation électrique : multi-dimensionnalité et bivalence, Décisions marketing, 83, 5-28.

Lutzenhiser L. (1992), A Cultural Model of Household Energy Consumption, Energy, $17,1,47-60$.

Moloney S. et Strengers Y. (2014), Going green ? : The limitations of behaviour change programmes as a policy response to escalating resource consumption, Environmental Policy and Governance, 24, 2, 94-107.

Özçağlar-Toulouse N. (2009), Quel sens les consommateurs responsables donnent-ils à leur consommation? Une approche par les récits de vie, Recherche et Applications en Marketing, 24, 3, 3-23.

Pallack M. et Cummings W. (1976), Commitment and voluntary energy conservation, Personnality and Social Psychology Bulletin, 2, 27-30.

Reckwitz A. (2002), Toward a theory of social practices: A development in culturalist theorizing, European Journal of Social Theory, 5, 2, 243-263.

Schatzki T.R. (1996), Social Practices. A Wittgensteinian approach to human activity and the social, Cambridge, Cambridge University Press.

Schatzki T.R. (2002), The site of the social: A philosophical account of the constitution of social life and change, University Park, Pennsylvania State University Press.

Shove E. (2003), Comfort, cleanliness and convenience. The social organization of normality, Oxford and New York, Berg.

Shove E. (2010), Beyond the ABC : Climate Change Policy and Theories of Social Change, Environment and Planing A, 42, 6, 1273-1285.

Shove E. et Pantzar M. (2005), Consumers, producers and practices, Journal of Consumer Culture, 5, 1, 43-64.

Spurling N., McMeekin A., Shove E., Southerton D. et Welch D. (2013), Interventions in practice: re-framing policy approaches to consumer behaviour, Sustainable Practices Research Group Report.

Stephenson J., Barton B., Carrington G., Gnoth D., Lawson R. et Thorsnes P. (2010), Energy cultures : a frame work for understanding energy behaviours, Energy Policy, $38,10,6120-6129$.

Thaler R.H. et Sunstein C.R. (2008), Nudge: Improving decisions about health, wealth, and happiness, New Haven, Yale University Press.

Warde A. (2005), Consumption and theories of practice, Journal of Consumer Culture, 5 , 2, 131-153.

Wilson C. et Dowlatabadi H. (2007), Models of Decision Making and Residential Energy Use, Annual Review of Environmental Resources, 32, 2, 1-35. 
Zhao T., Bell L., Horner M.W., Sulik J. et Zhang J. (2012), Consumer responses towards home energy financial incentives : A survey-based study, Energy Policy, 47, 8, 291297.

Zélem M-C. (2010), Politique de maîtrise de la demande d'énergie et résistances au changement. Une approche socio-anthropologique, Paris, L'Harmattan, coll. « Logiques sociales ». 
Annexe 1. Caractéristiques sociodémographiques des 40 répondants

\begin{tabular}{|c|c|c|c|c|c|c|c|c|}
\hline $\begin{array}{r}\text { Caractéristiques } \\
\text { du répondant } \\
\text { et du ménage }\end{array}$ & $\begin{array}{l}\text { Age du } \\
\text { répondant }\end{array}$ & $\begin{array}{l}\text { Genre du } \\
\text { répondant }\end{array}$ & $\begin{array}{l}\text { Age le plus } \\
\text { élevé dans } \\
\text { le ménage }\end{array}$ & $\begin{array}{l}\text { Adultes } \\
\text { (nombre) }\end{array}$ & $\begin{array}{l}\text { Enfants } \\
\text { mineurs } \\
\text { (nombre }\end{array}$ & $\begin{array}{l}\text { Personnes en } \\
\text { activité } \\
\text { professionnelle } \\
\text { (nombre) }\end{array}$ & $\begin{array}{l}\text { Niveau de formation } \\
\text { du répondant }\end{array}$ & $\begin{array}{l}\text { Revenu annuel du } \\
\text { ménage (hors Aides } \\
\text { au logement) }\end{array}$ \\
\hline \multicolumn{9}{|l|}{ Répondant } \\
\hline Albert & 79 & $\mathrm{M}$ & 80 & 2 & 0 & 0 & $\mathrm{~V}:$ niveau CAP-BEP & 0 à $9400 €$ \\
\hline Aline & 42 & $\mathrm{~F}$ & 42 & 1 & 1 & $\geq 1$ & IV : niveau baccalauréat. & 0 à $9400 €$ \\
\hline Amira & 35 & $\mathrm{~F}$ & 42 & 2 & 4 & $\geq 1$ & VI : inférieur à BEP-CAP & $23751 €$ à $38750 €$ \\
\hline Baptiste & 42 & M & 43 & 2 & 2 & $\geq 1$ & V : niveau CAP-BEP & $15001 €$ à $23750 €$ \\
\hline Béatrice & 35 & $\mathrm{~F}$ & 39 & 2 & 2 & $\geq 1$ & III : niveau bac +2 & $23751 €$ à $38750 €$ \\
\hline Bernard & 46 & M & 85 & 2 & 0 & $\geq 1$ & VI : inférieur à BEP-CAP & $9401 €$ à $15000 €$ \\
\hline Bertrand & 70 & M & 70 & 1 & 0 & $\geq 1$ & V : niveau CAP-BEP & $15001 €$ à $23750 €$ \\
\hline Carl & 43 & M & 37 & 2 & 3 & $\geq 1$ & IV : niveau baccalauréat. & $15001 €$ à $23750 €$ \\
\hline Catherine & 80 & $\mathrm{~F}$ & 80 & 1 & 0 & 0 & $\mathrm{~V}$ : niveau CAP-BEP & 0 à $9400 €$ \\
\hline Daniel & 80 & M & 80 & 1 & 0 & 0 & IV : niveau baccalauréat. & 0 à $9400 €$ \\
\hline Fabienne & 38 & $\mathrm{~F}$ & 38 & 1 & 3 & 0 & VI : inférieur à BEP-CAP & $9401 €$ à $15000 €$ \\
\hline Francine & 44 & $\mathrm{~F}$ & 44 & 2 & 0 & 0 & V : niveau CAP-BEP & 0 à $9400 €$ \\
\hline Françoise & 28 & $\mathrm{~F}$ & 28 & 1 & 1 & 0 & IV : niveau baccalauréat. & 0 à $9400 €$ \\
\hline Geneviève & 64 & $\mathrm{~F}$ & 64 & 1 & 0 & 0 & $\mathrm{~V}$ : niveau CAP-BEP & 0 à $9400 €$ \\
\hline Georgette & 72 & $\mathrm{~F}$ & 73 & 3 & 0 & 0 & VI : inférieur à BEP-CAP & $9401 €$ à $15000 €$ \\
\hline Jacqueline & 55 & $\mathrm{~F}$ & 65 & 2 & 0 & $\geq 1$ & IV : niveau baccalauréat. & $23751 €$ à $38750 €$ \\
\hline Josette & 64 & $\mathrm{~F}$ & 73 & 2 & 0 & 0 & $\mathrm{~V}$ : niveau CAP-BEP & $23751 €$ à $38750 €$ \\
\hline Laurence & 45 & $\mathrm{~F}$ & 82 & 2 & 0 & 0 & VI : inférieur à BEP-CAP & $15001 €$ à $23750 €$ \\
\hline Lucette & 64 & $\mathrm{~F}$ & 64 & 1 & 0 & 0 & VI : inférieur à BEP-CAP & 0 à $9400 €$ \\
\hline Madona & 20 & $\mathrm{~F}$ & 60 & 5 & 3 & $?$ & VI : inférieur à BEP-CAP & $?$ \\
\hline Mahmoud & 52 & M & 52 & 2 & 2 & $\geq 1$ & V : niveau CAP-BEP & $15001 €$ à $23750 €$ \\
\hline Martine & 48 & $\mathrm{~F}$ & 48 & 1 & 2 & 0 & VI : inférieur à BEP-CAP & 0 à $9400 €$ \\
\hline Maya & 63 & $\mathrm{~F}$ & 63 & 1 & 0 & 0 & VI : inférieur à BEP-CAP & 0 à $9400 €$ \\
\hline Melania & 42 & $\mathrm{~F}$ & 50 & 2 & 2 & $\geq 1$ & III : niveau bac+2 & $16001 €$ à $23750 €$ \\
\hline Michael & 28 & M & 28 & 2 & 0 & $\geq 1$ & $\mathrm{~V}$ : niveau CAP-BEP & $9401 €$ à $15000 €$ \\
\hline Mila & 37 & $\mathrm{~F}$ & 43 & 2 & 3 & $\geq 1$ & VI : inférieur à BEP-CAP & $9401 €$ à $15000 €$ \\
\hline Moktar & 51 & M & 51 & 2 & 5 & $\geq 1$ & V : niveau CAP-BEP & $15001 €$ à $23750 €$ \\
\hline Murielle & 57 & $\mathrm{~F}$ & 61 & 2 & 0 & 0 & $\mathrm{~V}$ : niveau CAP-BEP & $9401 €$ à $15000 €$ \\
\hline Nuria & 39 & $\mathrm{~F}$ & 44 & 2 & 5 & $\geq 1$ & $\mathrm{VI}$ : inférieur à BEP-CAP & $15001 €$ à $23750 €$ \\
\hline Ornella & 59 & $\mathrm{~F}$ & 59 & 3 & 0 & $\geq 1$ & V : niveau CAP-BEP & $9401 €$ à $15000 €$ \\
\hline Pamela & 28 & $\mathrm{~F}$ & 28 & 2 & 2 & $\geq 1$ & $\mathrm{~V}$ : niveau CAP-BEP & $9401 €$ à $15000 €$ \\
\hline Paulette & 76 & $\mathrm{~F}$ & 76 & 2 & 0 & 0 & VI : inférieur à BEP-CAP & $15001 €$ à $23750 €$ \\
\hline Pierre & 51 & M & 51 & 2 & 0 & $\geq 1$ & V : niveau CAP-BEP & $15001 €$ à $23750 €$ \\
\hline Régine & 50 & $\mathrm{~F}$ & 50 & 1 & 0 & 0 & VI : inférieur à BEP-CAP & 0 à $9400 €$ \\
\hline Reine & 53 & $\mathrm{~F}$ & 84 & 2 & 0 & 0 & VI : inférieur à BEP-CAP & 0 à $9400 €$ \\
\hline René & 65 & M & 65 & 1 & 0 & 0 & VI : inférieur à BEP-CAP & 0 à $9400 €$ \\
\hline Rihana & 26 & $\mathrm{~F}$ & 26 & 1 & 1 & 1 & V : niveau CAP-BEP & $9401 €$ à $15000 €$ \\
\hline Roger & 90 & M & 90 & 1 & 0 & 0 & $\mathrm{~V}$ : niveau CAP-BEP & $9401 €$ à $15000 €$ \\
\hline Rose & 27 & $\mathrm{~F}$ & 27 & 1 & 0 & $\geq 1$ & $\mathrm{~V}$ : niveau CAP-BEP & 0 à $9400 €$ \\
\hline Sophie & 55 & $\mathrm{~F}$ & 63 & 2 & 0 & 0 & VI : inférieur à BEP-CAP & $9401 €$ à $15000 €$ \\
\hline
\end{tabular}


Annexe 2. Adaptation de la grille théorique de compréhension des pratiques (GramHanssen, 2008) à une population de locataires de l'habitat social réhabilité thermiquement et présentation des quatre profils-types

\begin{tabular}{|c|c|c|c|c|c|c|c|}
\hline $\begin{array}{l}\text { Dimensions } \\
\text { d'appréhension } \\
\text { des pratiques }\end{array}$ & Définitions & $\begin{array}{c}\text { Composantes issues des discours des } \\
\text { répondants }\end{array}$ & $\begin{array}{c}\text { Comportements } \\
\text { de } \\
\text { consommation } \\
\text { concernés } \\
\end{array}$ & Volontaire & Responsable & Empêché & Réfractaire \\
\hline \multirow{3}{*}{$\begin{array}{l}\text { Compréhension } \\
\text { pratique du } \\
\text { monde } \\
\text { (Practical } \\
\text { understandings) }\end{array}$} & \multirow{3}{*}{$\begin{array}{l}\text { Activités physiques, } \\
\text { incorporées et routinières } \\
\text { (Schatzki, 1996; } \\
\text { Reckwitz, 2002) }\end{array}$} & $\begin{array}{l}\text { Modes d'utilisation des robinets } \\
\text { thermostatiques }\end{array}$ & \multirow{7}{*}{$\begin{array}{l}\text { Acquisition de } \\
\text { matériels }\end{array}$} & & & & \\
\hline & & Gestion de la veille des appareils & & & & & \\
\hline & & $\begin{array}{l}\text { Repères d'action incorporés par rapport à } \\
\text { l'histoire de vie (Habitudes de chauffage, } \\
\text { d'éclairage, de gestion des appareils) }\end{array}$ & & & & & \\
\hline \multirow{4}{*}{$\begin{array}{l}\text { Intelligibilité } \\
\text { pratique } \\
\text { (Practical } \\
\text { intelligibility) }\end{array}$} & \multirow{4}{*}{$\begin{array}{l}\text { Ce qui fait sens pour } \\
\text { l'individu, ce qui guide } \\
\text { ses pratiques (Schatzki, } \\
\text { 1996) }\end{array}$} & $\begin{array}{l}\text { Conserver son niveau de vie actuel et ne pas } \\
\text { régresser }\end{array}$ & & & & & \\
\hline & & $\begin{array}{l}\text { Conserver (et si possible développer) un } \\
\text { niveau de confort, de paraître et d'avoir au } \\
\text { moins aussi élevé que les autres }\end{array}$ & & & & & \\
\hline & & Faire des économies & & & & & \\
\hline & & $\begin{array}{l}\text { Penser par altruisme à ceux qui n'ont pas } \\
\text { accès à l'énergie ou au confort }\end{array}$ & & & & & \\
\hline \multirow{9}{*}{$\begin{array}{l}\text { Règles, savoirs, } \\
\text { langage } \\
\text { (Rules, } \\
\text { Knowlege, } \\
\text { langage) }\end{array}$} & \multirow{5}{*}{$\begin{array}{l}\text { Règles explicites, } \\
\text { principes, préceptes et } \\
\text { instructions d'usages } \\
\text { (Schatzki, 1996) }\end{array}$} & $\begin{array}{l}\text { Prescriptions : consignes données par le } \\
\text { bailleur social après la réhabilitation } \\
\text { thermique des logements }\end{array}$ & \multirow{9}{*}{$\begin{array}{l}\text { Usages des } \\
\text { équipements } \\
\text { (acquis et } \\
\text { incorporés au } \\
\text { logement) }\end{array}$} & & & & \\
\hline & & $\begin{array}{l}\text { incitations des médias à réduire sa } \\
\text { consommation d'énergie }\end{array}$ & & & & & \\
\hline & & $\begin{array}{l}\text { influence des tiers identifiés (proches en qui } \\
\text { on a confiance) }\end{array}$ & & & & & \\
\hline & & $\begin{array}{l}\text { influence des tiers non identifiés (vendeurs, } \\
\ldots \text { ) }\end{array}$ & & & & & \\
\hline & & $\begin{array}{l}\text { - Informations : quantitatives en lien avec la } \\
\text { consommation d'énergie (consommation } \\
\text { réalisée) }\end{array}$ & & & & & \\
\hline & \multirow{4}{*}{$\begin{array}{l}\text { Savoirs tacites (Shove et } \\
\text { Pantzar, 2005) }\end{array}$} & $\begin{array}{l}\text { quantitatives en lien avec la consommation } \\
\text { prévisionnelle d'énergie (étiquetage } \\
\text { énergétique) }\end{array}$ & & & & & \\
\hline & & $\begin{array}{l}\text {. économiques (fournies sur les relevés du } \\
\text { bailleur social) }\end{array}$ & & & & & \\
\hline & & $\begin{array}{l}\text {. économiques (fournies sur les factures des } \\
\text { fournisseurs) }\end{array}$ & & & & & \\
\hline & & $\begin{array}{l}\text { physiques (témoins de veille ou de } \\
\text { consommation) }\end{array}$ & & & & & \\
\hline \multirow{6}{*}{$\begin{array}{l}\text { Engagements et } \\
\text { significations } \\
\text { (Engagements } \\
\text { \& Meanings) }\end{array}$} & \multirow{6}{*}{$\begin{array}{l}\text { Fins, projets, tâches, } \\
\text { buts, croyances, } \\
\text { émotions, humeurs } \\
\text { (structures téléo- } \\
\text { affectives, Schatzki, } \\
1996,89 \text { ) }\end{array}$} & . Niveau de confort souhaité & $\begin{array}{l}\text { substitution/ } \\
\text { réduction de la }\end{array}$ & & & & \\
\hline & & $\begin{array}{l}\text { Image de soi recherchée au travers des } \\
\text { équipements }\end{array}$ & $\begin{array}{l}\text { consommation } \\
\text { énergétique }\end{array}$ & & & & \\
\hline & & $\begin{array}{l}\text { Volonté d'ouverture vers l'extérieur (sortir } \\
\text { pour faire du sport, échapper au cadre du } \\
\text { logement,) }\end{array}$ & & & & & \\
\hline & & $\begin{array}{l}\text { Volonté d'ouverture vers l'extérieur } \\
\text { (rencontrer d'autres personnes) }\end{array}$ & & & & & \\
\hline & & $\begin{array}{l}\text { Faire soi-même pour réduire la } \\
\text { consommation }\end{array}$ & & & & & \\
\hline & & $\begin{array}{l}\text { Inciter les autres à réduire leur } \\
\text { consommation }\end{array}$ & & & & & \\
\hline \multirow{2}{*}{$\begin{array}{l}\text { Eléments } \\
\text { matériels } \\
\text { (The material } \\
\text { éléments) }\end{array}$} & \multirow{2}{*}{$\begin{array}{l}\text { Choses (Reckwitz, 2002) } \\
\text { Produits et technologies } \\
\text { (Shove et Pantzar, 2005) } \\
\text { Objets de consommation } \\
\text { (Warde, 2005) }\end{array}$} & $\begin{array}{l}\text { Système incorporé : ensemble comprenant } \\
\text { isolation, chaudière, huisserie, radiateurs,... }\end{array}$ & & & & & \\
\hline & & $\begin{array}{lrr}\text { Equipements } & \text { rapportés }: & \text { achats } \\
\text { d'électroménager, de matériels audiovisuels, } \\
\text { électroniques, } \\
\text { communication }\end{array}$ & & & & & \\
\hline
\end{tabular}

Composante favorable à la réduction de la consommation d'énergie Composante défavorable à la réduction de la consommation d'énergie Item sans expression d'orientation spécifique 